\title{
El legado de Sabine MacCormack en los Andes
}

\author{
Víctor MaQque
}

\section{RESUMEN}

La doctora Sabine MacCormack, una historiadora de estudios clásicos y andinos, ha legado su extraordinaria biblioteca al Instituto de Pastoral Andina (IPA) en el Perú. La profesora Sabine fue reconocida por su labor académica ejemplar, por sus imprescindibles libros y numerosos artículos sobre la compleja historia colonial de los Andes. Su trayectoria de Alemania al Reino Unido y luego a los Estados Unidos ha sido tan significativa como la magnitud de sus investigaciones del mundo clásico grecorromano y la Latinoamérica colonial. Sus colegas y amigos, reflexionando sobre su trágico fallecimiento, han coincidido en la importancia de un examen intelectual exhaustivo de mundos aparentemente desconectados como el que Sabine llevó a cabo. Los aportes de Sabine, sin embargo, continúan vibrantes en los numerosos seguidores de sus estudios y en la colosal biblioteca personal que con tanto esmero atesoró y organizó y que ahora se encuentra en el Perú. 
Palabras clave: Sabine MacCormack, Andes coloniales, Historia Clásica Grecorromana, Arte del historiador

\begin{abstract}
Dr. Sabine MacCormack, a historian of Classics and of the Andes, left her extraordinary library to the Instituto Pastoral Andina (IPA) in Perú. Professor MacCormack was recognized by her exemplary scholarship, her books, and numerous articles examining the complex history of colonial Andes. Her trajectory from Germany to England, and then to the United States was as significant as the scope of her studies from the classic Greco-Roman to the Andean world. Her colleagues and friends on mourning her tragic death reflected on the importance of a comprehensive intellectual inquiry into the seemingly detached societies that MacCormack studied. The contributions of MacCormack, however, continue thriving on the number of followers of her studies and her carefully collected and curated library that is now located in Peru.
\end{abstract}

Keywords: Sabine MacCormack, colonial Andes, Classic Greco-Roman History, Historian's Craft

HACE UNA DÉCADA conocí a la profesora Sabine en dos conversaciones aparentemente contradictorias. Por una generosa invitación me hallaba en la Universidad de Notre Dame (Indiana, USA), al terminar mi presentación, un profesor que vio algunas coincidencias con temas de una investigadora amiga suya en la universidad, me sugirió conversar con la profesora Sabine MacCormack (Frankfurt, 1941 - South Bend, 2012). Seguí la sugerencia y toqué la puerta de su oficina, me presenté y Sabine me recibió muy amablemente. Me habló algunas palabras en quechua a lo que respondí con agrado y luego en un fluido ir y venir entre el castellano y el inglés, me describió con gran entusiasmo el programa del doctorado en historia de América Latina que ella lideraba. Me animó a postular, me contó de las bondades del programa, los cursos, la calidad de los profesores 
y, por supuesto, lo que picó más mi curiosidad, las oportunidades de becas. Rápidamente, sin embargo, me pidió que volviera al día siguiente para mostrarme en detalle los contenidos de los cursos, pues en ese momento ella debía salir a una reunión. Cuando volví al siguiente día, Sabine tenía una actitud sorprendentemente opuesta a la del día anterior, me dijo que el programa era nuevo, más bien pequeño y que no me convenía. Dijo que yo debería postular a los programas de doctorado en las universidades de Wisconsin, Michigan o California, pues esas estaban, según ella, más avanzadas en sus especialidades de América Latina. Quedé intrigado por el contraste entre ambas conversaciones.

Sabine MacCormack, en ese entonces, era la profesora distinguida con la cátedra Fr. Theodore M. Hesburgh, CSC. en Notre Dame. Sus estudios y publicaciones eran considerados de una exuberante erudición y son lecturas obligadas en la academia del hemisferio norte. Sabine tenía una comprensión histórica impresionante, fue una reconocida experta como historiadora del mundo clásico grecorromano, así como también del mundo colonial andino. Según una nota póstuma de Matthew Reisz en el Times Higher Education, su carrera académica fue creciendo en el tiempo (Reisz 05.07.2012). Obtuvo su doctorado en historia en la Universidad de Oxford, en 1974, y con mucha laboriosidad se abrió campo en la disciplina. Trabajó como instructora asistente en la Universidad de Sidney, también como bibliotecaria, archivista, traductora y luego como maestra. En 1979 consiguió su primer empleo en Estados Unidos como profesora de historia en la Universidad of Texas en Austin, luego como profesora de estudios clásicos e historia en la Universidad de Stanford (1982), Michigan (1990) y, desde 2004 hasta su fallecimiento en 2012, como profesora distinguida en la Universidad de Notre Dame, en Indiana.

Sus publicaciones son enciclopédicas. Aquí solamente enumero sus libros como una muestra de la importancia de sus aportes: Concise Enciclopedia of Greek and Roman Mythology (publicada, con su 
apellido de soltera Sabine G. Oswalt, por Collins 1969), Art and Ceremony in Late Antiquity (University of California Press, 1981), The Shadows of Poetry: Vergil in the Mind of Augustine (Berkeley, University of California Press, 1998), Religion in the Andes: Vision and Imagination in Early Colonial Peru (Princeton University Press, 1991) y On the Wings of Time Rome, the Incas, and Spain (Princeton University Press, 1991). De acuerdo a la revista Perspectives on History, los influyentes artículos que Sabine publicó suman más de 60 en las revistas más importantes en la disciplina (Ando y Lizz 2013). Su exitosa labor de enseñanza y sus publicaciones fueron reconocidas con títulos honoríficos y premios. En la Universidad de Michigan recibió la cátedra de profesor titulada Alice Freeman Palmer y en Notre Dame, el honorable título del legendario rector, el padre Theodore Hesburgh, CSC. Su libro On the Wings of Time recibió dos premios, el James A. Rawley y el John Edwin Fagg de la Asociación de Historiadores en los Estados Unidos (AHA, por sus siglas en Inglés). Entre otros importantes reconocimientos, Sabine recibió el Dumbarton Oaks Fellowship, también fue elegida fellow del Medieval Academy of America, de la American Philosophical Society, la American Academy of Arts and Sciences y la codiciada distinción de Mellon Professorship en Historia de América Latina.

Los dos encuentros iniciales, aunque contradictorios en cierto modo, me hicieron decidir a postular a Notre Dame. Al año siguiente ya estaba sentado en el curso The Historian's Craft que Sabine enseñaba. Había preparado un curso enciclopédico, extenso en su cobertura y profundo en sus contenidos, un estudio a fondo de los académicos que ella consideraba más influyentes en la disciplina. Un examen exhaustivo de los escritos más importantes de historiadores clásicos del hemisferio norte, como Heródoto, Tucídides, Marc Bloch, Arnaldo Momigliano, Anthony Grafton, J. H. Elliot, Amos Funkenstein, Ibn Khaldun, entre otros. Sabine describía el contexto social de los autores, examinaba (con algunas intervenciones nuestras) el método de estos autores, e inmediatamente presen- 
taba una charla magistral sobre el proceso, las dificultades y aportes del autor a la disciplina histórica. La metodología y la dinámica del curso, aunque impresionante a veces, casi no dejaba tiempo para la intervención y discusión de estudiantes, lo que no gustó a todos en la clase. Sabine, sin embargo, con su amplio dominio de los textos asignados siempre combinados con ejemplos de sus propias investigaciones, algunos excelentes escritos y su voz firme, disuadió cualquier conato de malestar o queja en la clase.

Sabine promovía un encuentro de los estudiantes con estos autores selectos para examinar los retos fundamentales de la profesión histórica. Por ejemplo, nos invitó a escudriñar los trabajos de Heródoto de Halicarnaso (considerado el padre de la Historia) especialmente las interpretaciones históricas de las percepciones que la sociedad de la antigua Grecia tenía sobre la intervención divina en la vida cotidiana. Sabine era una experta en la religiosidad en las sociedades clásicas tanto europeas como andinas. Sus textos Art and Ceremony in Late Antiquity y Religion in the Andes dan testimonio de ello. Asimismo, revisamos los trabajos de Tucídides, crítico de Heródoto, quien postulaba una historia más en sentido de causa-efecto, una historia política de hechos recientes desacreditando como ilegítima la historización de eventos de los que el autor no ha sido testigo. Según Sabine, Tucídides tenía la idea de que la única historia seria era resultado de las historizaciones de hechos observados y de pensamientos que podían ser descritos sin filtros. Igualmente, revisamos las increíbles contribuciones de Marc Bloch, el gran historiador y soldado francés que, en plena segunda guerra mundial, argumentaba que el mayor enemigo del historiador era pretender juzgar los hechos históricos. Bloch fue el creador de la escuela y revista especializada Annales que, como escribe George Huppert, desde 1929 publicaba todo lo nuevo, lo dinámico y controversial en la literatura histórica francesa.

El examen exhaustivo de un tema con la máxima rigurosidad posible era para Sabine un requisito vital para quien intentara en serio 
dedicarse a la disciplina histórica. Para mi primer ensayo en su curso planteé a Sabine que planeaba un examen del método histórico en La Nueva Coronica y Buen Gobierno y Los Comentarios Reales de los Incas, en comparación al método en las obras de Heródoto y Tucídides. Su primera pregunta fue: «¿Has leído los trabajos de ambos autores andinos?». «No todo, pero tengo un buen entendimiento de sus obras», fue mi respuesta, revelando ingenuamente mi reciente transición de la incipiente academia de Puno al sistema académico norteamericano. Sabine me miró casi con molestia y sentenció: «Si no has leído las dos obras completas de principio a fin, no has leído nada». Por la fuerza en su mirada, más que por la de su voz y sus gentos al hablar, asentí y me dirigí inmediatamente a la biblioteca en busca de los textos de Guamán Poma y del Inca Garcilaso de la Vega.

La última vez que hablé con ella fue en la reunión de LASA 2012, en la ciudad de San Francisco, California (un mes antes de su fallecimiento). Vino a escuchar el panel en el que yo presenté un ensayo reciente; fiel a su estilo, se sentó en la primera fila, justo frente a mí. Al terminar, me preguntó con mucho interés sobre el origen de los documentos que cité. Mencioné que provenían del Archivo Regional de Puno, que estaban en condiciones de conservación precarias y que, sin embargo, había podido copiar una gran mayoría de ellos. Ella, firme en su visión, aseveró que «no es suficiente, hace falta copiar todo». Sugirió alianzas con personas e instituciones de su entorno que podrían cooperar y acordamos una siguiente reunión con resultados de las gestiones, sin embargo, su partida suspendió abruptamente este proyecto.

Sabine tenía una dinamicidad intelectual con características de tempestad en el mundo académico y a nivel personal. La cantidad de eventos académicos dentro y fuera del campus en que estaba involucrada era fenomenal. Aparte de las clases que dictaba, su agenda estaba repleta de participaciones en conversatorios especializados, seminarios y conferencias. Insistía en que por lo menos una vez al año debía haber en el campus un evento académico re- 
lacionado a los Andes. Siguiendo su sugerencia, organizamos una conferencia y al seleccionar nombres de expertos como invitados especiales nos preguntábamos si aceptarían nuestra invitación. Sabine, que seguía nuestros esfuerzos, a veces intervenía para decir: «déjenme darle una llamada esta noche para ver como esta su agenda y ver si puedo convencerlo a venir». Casi siempre, ponía su casa a nuestra disposición, así proveía un amplio espacio para conversaciones fructíferas, comida abundante y siempre un bar abierto bien surtido.

Para quienes tuvimos la oportunidad de interactuar con Sabine, su casa ha sido siempre el lugar de encuentros significativos. Alrededor de su jardín, en la mesa, junto a la chimenea, o en su majestuosa biblioteca del sótano, las conversaciones eran interminables. Esas tertulias en su casa casi siempre tendían a centrarse sobre temas de su pasión, la historia y la política del mundo de la antigüedad clásica, Roma, Grecia, España y los Andes. En su casa uno encontraba siempre intelectuales, estudiantes, y personalidades de lugares diversos: Gary Urton (Harvard), Bruce Manheim (Universidad de Michigan), Rolena Adorno (Yale), Mathew Restall (Penn State University), Alan Durston (Universidad de York, Canadá), Felipe Fernández-Armesto (Oxford y Notre Dame) y Ramón Mujica (Biblioteca Nacional del Peru) fueron algunos a quienes tuve la oportunidad de conocer o reencontrame y continuar diálogos y amistad. La comida era presentada siempre con mucho detalle, mientras que las bebidas, sobre todo el vino y el queso, parecían allí ser fruto del milagro de la multiplicación. Sin embargo, en ocasiones especiales, ella insistía en tener una buena botella de pisco peruano. Recuerdo que una vez, al inicio de una reunión, Sabine, al darse cuenta que no tenía pisco en el bar, con mucha ansiedad envió a un taxista conocido a comprar pisco a Chicago (a dos horas de distancia). Su determinación por tener pisco para compartir en dicha ocasión mostraba el cariño que tenía por el Perú, un país que, como decía ella, le había acogido en su seno, en el Cusco. 
Sabine contaba con entusiasmo sus experiencias de investigación y residencia en Perú: Cusco y Lima. Sus experiencias en Perú, especialmente su residencia por dos años en Cusco, fueron, por su puesto, a veces tempestuosas. Ella tenía una sensibilidad única sobre la preservación y el acceso a los bienes patrimoniales y archivísticos. Durante sus incansables horas de investigación en el Archivo Regional del Cusco (ARC), el Archivo General de la Nación, la Biblioteca Nacional en Lima, los archivos eclesiales, así como algunos archivos y bibliotecas particulares, observó con acuciosidad no solo los contenidos sino también el estado de conservación de los documentos. Recordaba con admiración cuando veía que las personas se esforzaban por una buena organización y mantenimiento de estas colecciones, y se quejaba enérgicamente cuando encontraba lo contrario. Contaba con malestar cómo una vez el empleado de un archivo peruano apareció frente a su hotel, ofreciéndole en venta una colección de documentos que había estado examinando por varios días. Ella aseguraba que le dio al empleado una lección de cómo ese acto era una traición a su país, sin embargo, no estaba segura que el mensaje fuera bien recibido.

Más allá de estas dificultades, estableció no solo su acceso sino su presencia en estos lugares. Los empleados del ARC, por ejemplo, sabían dónde se sentaba la doctora Sabine cuando estaba de visita, un empleado me contó una vez que aún los usuarios lo sabían. Ella era sin duda una de los visitantes que más tiempo y fondos invirtió en ese archivo, pues adquirió copias de colecciones enteras de muchos expedientes. Los voluminosos paquetes de copias fotostáticas con los sellos de estos repositorios ocupaban una sección importante de su biblioteca personal. Muchas de estas copias sobresalían en los estantes de su sótano por los tamaños enormes de papel en que estaban copiados.

Sabine había establecido en el hemisferio norte su reputación como una de las mayores autoridades en la historia colonial de los Andes y era así también reconocida en Perú. Sus visitas a Lima, por 
ende, eran conocidas en el círculo de académicos y fue varias veces invitada especial en eventos sobre los Andes. Sus intervenciones en estos eventos eran conocidas por su gran nivel y también a veces por la severidad de sus observaciones. No callaba cuando consideraba que algunos estaban equivocados o les faltaba rigurosidad. «Muchos que se precian de ser académicos en Perú y Latinoamérica son muy provincialistas y algunos no son más que plagiadores de tesis», me dijo una vez. Es más, continuó, «no devuelven libros prestados, por el contrario los destrozan escribiendo, doblando o rompiendo las páginas». Así, algunas de sus críticas le granjearon resentimientos que perduraron. No obstante, tenía una relación cercana con algunos académicos en el Perú, sobre todo profesores de la Pontificia Universidad Católica del Perú, en Lima. Apreciaba, por ejemplo, a Franklin Pease. Decía ella que era un amigo entrañable y un historiador de talla mundial, por su capacidad de entrar en diálogo con académicos a nivel internacional.

Junto con la historia de los Andes, Sabine tenía predilección por los paisajes andinos. Pintó y fotografió cada lugar que visitó en los valles y montañas de los Andes. Los que conocían bien a Sabine, sabían que cuando ella estaba en los Andes, además de sus documentos personales, en su bolso llevaba siempre una cámara fotográfica, sus pinceles y unas telas para pintar. Sharon Schierling, directora administrativa del Kellogg Institute en la Universidad de Notre Dame, cuenta una anécdota que revela la pasión de Sabine por los paisajes andinos. Una mañana ambas llevaban prisa por visitar un lugar arqueológico, por tanto, tomaron un taxi del Cusco para llegar y volver a tiempo para otros compromisos. En el camino, Sabine iba explicando los nombres de los cerros circundantes y, de pronto, pidió al taxista detenerse. Sabine se apeó del vehículo, bajó hacia una pequeña planicie en la ladera frente a una vista espectacular de un valle, sacó sus utensilios y, olvidando completamente la prisa que llevaban, se sentó a pintar. Para una muestra de su talento pictórico, su libro On the Wings of Time contiene algunas de sus obras, incluida una exquisita pintura en la tapa. 
Sabine fue una ávida lectora y coleccionista de libros y formó una magnífica biblioteca personal. Su colección incluyó primeras ediciones de textos históricos clásicos, de teología, literatura especializada y muchas copias de manuscritos y documentos de archivos importantes como el Archivo de Indias, AGN de Lima, ARC, ABNB Sucre, entre otros. En conversaciones personales, Sabine expresaba su voluntad de que, cuando llegara el momento, le gustaría donar su biblioteca al Instituto de Pastoral Andina (IPA) en Cusco. Decía que durante su estadía y numerosas visitas a Cusco, uno de sus lugares favoritos de estudio era la biblioteca del IPA, ubicada en la urbanización Santa Mónica en Wanchaq, Cusco. Sabine admiraba la larga historia de trabajo académico y pastoral del IPA en los Andes del Perú y tenía confianza en que su biblioteca sería usada y cuidada adecuadamente. Algunos amigos de Sabine la oyeron decir alguna vez que también había mencionado la biblioteca de la PUCP en Lima o la misma biblioteca de la Universidad de Notre Dame como destinatarios de su colección. Sin embargo, una vez más, ella había expresado su preferencia por los Andes y decidió donarlo al IPA, así lo hizo saber la única heredera, su hija Catherine. El entonces director del IPA, Fernando Valle Rondón, ha dirigido la complicada tarea de organizar, empacar y trasladar la colección desde el sótano de la casa de Sabine en South Bend (Indiana) hasta Arequipa. Después de una eficaz labor, la colección ahora nombrada biblioteca Sabine MacCormack fue presentada como parte de la biblioteca de la Universidad Católica San Pablo de Arequipa. Según la publicación oficial del IPA, la biblioteca MacCormack cuenta con una colección de 12,180 registros y se halla ahí por cesión temporal de diez años mientras el IPA organiza un lugar adecuado para la colección (IPA s/f).

Los esfuerzos en aprender y promover el estudio y enseñanza del quechua fueron otro aspecto de su extenso y profundo interés por los Andes. Durante su residencia en Cusco, recibió clases de quechua y disfrutaba las oportunidades que tenía de poner en práctica lo aprendido. Cada vez que pudo, convenció a sus buenos 
amigos cusqueños Donato Amado, Gina Maldonado e Inés Callalli a organizar visitas a alguna comunidad para conocer y conversar con las artesanas. Como suele ocurrir, las artesanas a veces le obsequiaban algunas canciones en quechua, Sabine en retribución se animaba a cantarles en alemán, su idioma materno. En la Universidad de Michigan, apoyó la enseñanza del idioma nativo más hablado en los Andes. Cuando se trasladó a la Universidad de Notre Dame en Indiana, hizo gestiones para crear el programa llamado Latin American Indigenous Languages Learning Program y que en él se enseñara el quechua. Para ello invitó a dos de sus profesoras del Cusco que se turnaban anualmente para enseñar. También dedicó los fondos de su premio de la Fundación Andrew H. Mellon para la sostenibilidad del curso de quechua en Notre Dame. En los tres años pasados, respaldado por el Instituto Kellogg, he tenido el honor de desarrollar el programa de acuerdo con los estándares académicos exigidos y enseñar diversos niveles del curso de Lengua y Cultura Quechuas. Con los materiales de enseñanza producidos por el Instituto de Idiomas Maryknoll en Cochabamba (Bolivia) y la participación del Centro Tinku en Cusco, estudiantes interesados en los Andes desde disciplinas como literatura, teología, pre-medicina, antropología, historia, ciencias políticas e ingenierías han tenido la oportunidad de aprender el quechua, hacer pasantías, y realizar investigaciones académicas en diversas partes de los Andes. El legado de Sabine es inmenso y se mantiene presente en quienes la conocieron y en los que la seguirán conociendo por medio de sus escritos y por su colección en manos del IPA.

La abrupta partida de Sabine, a sus dinámicos 71 años de edad, tomó por sorpresa a todos quienes la conocían. Las ceremonias y conmemoraciones en su honor fueron numerosas y muy sentidas. Para una muestra señalo aquí algunas que he visto. La misa en su memoria en la Basílica, como es conocida la iglesia en el campus de Notre Dame, estuvo abarrotada por sus colegas, estudiantes y amigos. El padre John Jenkins CSC., rector de la universidad, que 
presidió la misa, recordó con gratitud su contribución a la calidad académica y sus consejos, pues ella fue parte de su equipo de asesores. Scott Mainwaring, entonces director del Kellogg Institute, que era el centro de operaciones de Sabine, expresó que «fue uno de los profesores más distinguidos en Notre Dame» (Kellog Institute s/f). Sabine urgió un «estudio histórico responsable y completo de las poblaciones» en un espacio que fue latino y castellano, quechua y aimara, europeo y americano a las vez, reconocieron otros colegas en la revista Perspectives on History de la American Historical Association (Ando y Lizz 2013). La publicación Times Higher Education expresó: «Una historiadora de "energías exuberantes" que se sentía en casa en el mundo de la antigua Roma y la Latinoamérica colonial acaba de fallecer» (Reisz 2012). Las personas cercanas a Sabine interpretaron como un signo benigno que el paro cardíaco que se la llevó la encontró un día soleado de primavera laborando en el jardín de su casa, un jardín que ella cultivó y cuidó con el mismo esmero que su labor académica.

\section{REFERENCIAS}

Ando, Clifford y Peggy Lizz

2013 «Sabine MacCormack 1941-2012» En: Perspectives on History. Publicación electrónica [en línea], fecha de publicación: 08.2013. Disponible en: <https://www.historians.org/publicationsanddirectories/perspectives-on-history/september-2013/in-memoriam-sabine-maccormack $>$.

Instituto de Pastoral Andina (IPA)

s/f «Sabine MacCormack». En: Instituto de Pastoral Andina. Publicación electrónica [en línea]. Disponible en: <http://www.ipandina.org/ tag/biblioteca-sabine-maccormack/>. 
KeLLOG Institute

s/f «Memoriam Sabine MacCormack». En: Kellog Institute for International Studies. Publicación electrónica [en línea]. Disponible en: $<$ https://kellogg.nd.edu/memoriam-sabine-maccormack>.

ReISZ, Mathew

2012 «Sabine MacCormack, 1941-2012». En: Times Higher Education. Publicación electrónica [en línea], fecha de publicación: 5 de julio de 2012. Disponible en: <https://www.timeshighereducation.com/news/people/obituaries/sabine-maccormack-1941-2012/420438.article>. 